\title{
Upregulation of METTL3 Expression Predicts Poor Prognosis in Patients with Esophageal Squamous Cell Carcinoma
}

This article was published in the following Dove Press journal: Cancer Management and Research

\section{Tian-Liang Xia* Shu-Mei Yan (D* \\ Li Yuan \\ Mu-Sheng Zeng}

State Key Laboratory of Oncology in South China; Collaborative Innovation Center for Cancer Medicine; Sun Yat-sen University Cancer Center, Guangzhou, People's Republic of China

*These authors contributed equally to this work
Correspondence: Mu-Sheng Zeng Email zengmsh@sysucc.org.cn
Background: The $\mathrm{N}^{6}$-methyladenosine $\left(\mathrm{m}^{6} \mathrm{~A}\right)$ RNA modification of mRNA mediates various cellular functions and cancer progression. However, the roles of $\mathrm{m}^{6} \mathrm{~A}$ RNA modification in the regulation of esophageal squamous cell carcinoma (ESCC), the dominant subtype of esophageal cancer in Asia, were unclear.

Materials and Methods: Here, we analyzed the mRNA expression level of methyltransferase like 3 (METTL3) in the public available datasets of ESCC tissues and matched adjacent normal tissues. We also performed immunohistochemistry (IHC) assays to detect the protein expression of METTL3 in human ESCC tissue specimen. In our study, we also analyzed the association between METTL3 expression and prognosis using Cox proportional hazard regression in 207 ESCC patients.

Results: The results of public available datasets and IHC assays showed that METTL3 was upregulated in tumor compared with adjacent nonmalignant esophageal mucosal tissues. The IHC results indicated that higher expression level of METTL3 was associated with worse survival. We also found that METTL3 expression level was an independent predictor for disease-free survival and overall survival of ESCC patients.

Conclusion: Our results revealed that the METTL3 expression level could be used as an independent prognostic biomarker for ESCC prognosis.

Keywords: esophageal squamous cell carcinoma, METTL3, prognosis, survival

\section{Introduction}

$\mathrm{N}^{6}$-methyladenosine $\left(\mathrm{m}^{6} \mathrm{~A}\right)$ RNA modification, which was discovered in the $1970 \mathrm{~s}$, occurs across different regions of mRNA transcripts and is particularly enriched near the stop codons. The transcriptome-wide $\mathrm{m}^{6} \mathrm{~A}$ sequencing results suggest the consensus motif DRA* CH (D denotes A, G or U; R denotes $G$ or A; A* denotes methylatable $\mathrm{A}$; and $\mathrm{H}$ denotes $\mathrm{A}, \mathrm{C}$ or $\mathrm{U}) .{ }^{1-4}$ Many $\mathrm{m}^{6} \mathrm{~A}$-associated proteins have been identified by comprehensive and systematic mass-spectrometry-based screening of $\mathrm{m}^{6}$ A interactors. ${ }^{5}$ The "writer" heterotrimeric complex consists of the enzyme methyltransferase like 3 (METTL3) and the two co-factors called METTL14 and WTAP. ${ }^{6-9}$ The "eraser" proteins FTO and ALKBH5 are RNA demethylases. ${ }^{10,11}$ YTH domaincontaining family proteins (YTHDF1, YTHDF2, YTHDF3, YTHDC1 and YTHDC2) are the most studied "reader" proteins, which regulate RNA metabolism in many aspects. $^{12-18}$ Importantly, recent studies found that METTL3, having $\mathrm{m}^{6} \mathrm{~A}$ enzymatic activity, regulated various tumorigenesis. ${ }^{19-22}$ However, whether METTL3 could affect esophageal squamous cell carcinoma (ESCC) progression is unclear. 
Esophageal cancer is the 8th most common cancer and the 6th most common cause of cancer-related deaths. ${ }^{23}$ ESCC is the dominant subtype of esophageal cancer in Asia, accounting for $90 \%$ of the cases in China. ${ }^{24}$ A notably high incidence is observed in China. ${ }^{24}$ So far, no effective ESCC prognostic factors have been characterized, and the overall 5-year survival rate for ESCC is less than $15 \% .{ }^{25}$ It is worth to investigate whether METTL3 could regulate ESCC progression and whether it could be an effective predictor for ESCC prognosis.

Here, we detected the expression of METTL3 in a total of 207 ESCC patients by immunohistochemistry (IHC) assays and found that increased expression of METTL3 was associated with poor prognosis for ESCC patients.

\section{Materials and Methods}

\section{Human ESCC Tissue Specimens}

The Internal Review and Ethics Board of Sun Yat-sen University Cancer Center, Guangzhou, China approved this study. This study was conducted in accordance with the Declaration of Helsinki. Patients whose tissues and medical records were used for this study had provided written informed consent. This study included 207 ESCC patients, who were diagnosed from October 2000 to April 2007 in Sun Yat-sen University Cancer Center and underwent surgical resection without neoadjuvant/adjuvant therapy. We used the 7th edition of the TNM classification of the International Union Against Cancer (2009) to record the histologic grade and the clinical stage of the tumors. Patients' age, sex, tumor location, histopathology grade, histopathology tumor status, $\mathrm{N}$ categories, TNM stage were recorded by reviewing the patient medical records after surgery. Follow-up assessments were performed every 3 months postoperatively for the first years, and followed by every 6 months later.

\section{Immunohistochemistry Staining Analysis}

Total 207 ESCC specimens were selected for IHC staining to determine METTL3 expression. First, the 4- $\mu \mathrm{m}$ FFPE ESCC samples were placed in an oven for $2 \mathrm{~h}$ at $65^{\circ} \mathrm{C}$, and then deparaffinized in xylene and rehydrated with ethanol alcohol followed by distilled water. To block endogenous peroxidase activity, the sections were immersed in 3\% hydrogen peroxide at room temperature for $10 \mathrm{~min}$. After that, the sections were boiled in Citrate Antigen Retrieval Solution $(\mathrm{pH}=6.5)$ for $4 \mathrm{~min}$ in an electric pressure cooker for antigen retrieval. The sections were then incubated with rabbit monoclonal antibody against METTL3 (1:500 dilution; ab195352, Abcam) at $37^{\circ} \mathrm{C}$ for $1 \mathrm{~h}$ in a moist chamber and a goat anti-rabbit secondary antibody at $37^{\circ} \mathrm{C}$ for half an hour. Subsequently, the tissue sections were stained with DAKO Liquid 3,'3-diaminobenzidine tetrahydrochloride (DAB) for 10 min, counterstained with $10 \%$ Mayer's hematoxylin, then were dehydrated and mounted. Normal rabbit IgG was used as a negative control. Each section was independently evaluated by two pathologists.

The staining results were scored based on two criteria: (1) the proportion of tumor cells staining positive: 0 (none), 1 (1\% to $10 \%), 2$ (11\% to $50 \%), 3(51 \%$ to $75 \%)$, and 4 (76\% to $100 \%)$; and (2) the average intensity of the positive cells: 0 (none), 1 (weak), 2 (moderate) and 3 (strong). The proportion and intensity scores were then multiplied to obtain a total score (range, 0 to 12). All these patients were divided into two groups according to the median total score, and the score higher than 8 was considered as high METTL3 expression.

\section{Bioinformatic Analysis}

Differential expression analysis of METTL3 in ESCC tissues versus matched normal tissues was performed by using public available microarray datasets GSE23400, GSE38129 and GSE75241.

\section{Statistical Analysis}

Data analyses were performed using SPSS v-23 (IBM), R v3.3.3 and GraphPad Prism 6. The Student's $t$-test was used to compare different METTL3 expression between normal and ESCC groups. The change of METTL3 expression was showed with the $\log _{2} \mathrm{FC}$ (fold change of METTL3 expression from tumor to normal tissues). The Benjamini-Hochberg approach for the false discovery rate (FDR) was used for the multiple testing correction. We defined the results as statistical significant when the adjusted $p$-value $<0.05$. Chi-square test was used to determine the correlation between METTL3 expression and the clinicopathological variables. Disease-free survival was defined as the time from surgery to cancer progression or metastasis. Overall survival was defined as the time from surgery to death. Cumulative survival time was calculated using the Kaplan-Meier method, and compared using the Log-rank test. The effects of various clinicopathological features and METTL3 expression on survival were assessed using Cox proportional hazard regression 
analysis. For all analysis, $p$-value $<0.05$ indicates statistical significance.

\section{Results}

\section{Relationship Between METTL3 Expression and Clinicopathological Variables}

We analyzed METTL3 expression using three microarray datasets of ESCC tissues. We found that METTL3 mRNA expression level was significantly upregulated in ESCC tissues compared with matched adjacent normal tissues (Figure 1A-C). There are a few METTL3 downregulated samples, which might be caused by the effects of the individual differences. These results indicated that METTL3 has a potential oncogenic function for ESCC progression.

We then performed IHC assays in 207 ESCC tissues to detect the expression level of METTL3. We found that METTL3 was expressed in nuclei, shown as brown-yellow granules (Figure 1D-F). METTL3 was lower expressed in normal esophageal mucosa tissues (Figure 1D) than ESCC tissues (Figure 1E and F). We also found that METTL3 was higher expressed in ESCC tissues than adjacent nonmalignant esophageal mucosal tissues (Figure 1E and $\mathrm{F}$ ). The IHC samples were divided into two subgroups according to the expression level of METTL3: low METTL3 expression subgroup (Figure 1E) and high METTL3 expression subgroup (Figure 1F). Low expression level of METTL3 was detected in 128 of 207 ESCC tissues, whereas high expression level of METTL3 was detected in 79 of 207 ESCC tissues.

We then analyzed the correlation between METTL3 expression and patient clinicopathological variables. As summarized in Table 1, higher METTL3 expression was shown in patients with lymph node metastasis and advanced TNM stage ( $p=0.045$ and $p=0.004$, respectively). But METTL3 expression was not statistically correlated with age, gender, tumor location, histological grade or histopathology tumor status $(p=0.855,0.905,0.456$, 0.279 and 0.157 , respectively).

\section{Higher Expression Level of METTL3 Was Associated with Worse Survival}

Using Kaplan-Meier survival analysis (Log-rank test), we investigated the correlation between METTL3 expression and the survival status of ESCC patients. We found that patients with higher METTL3 expression experienced significantly worse overall survival (OS) $(p<0.001)$ and disease-free survival (DFS) $(p<0.001)$ compared with those with lower METTL3 expression level (Table 2, Figure 2A). The mean OS were 74.9 and 44 months in the low and high METTL3 expression groups, respectively (Table 2). The mean DFS were 72.4 and 38.6 months in the low and high METTL3 expression groups (Table 2). We further estimated the effect of METTL3 high expression on OS or DFS by subgroup analysis according to the histopathology tumor status, pT1-2 and pT3-4. We found that worse OS and DFS were observed in patients with higher METTL3 expression in pT3-4 $(p<0.001$ and $p<0.001$, respectively, Table 2), but not in pT1-2 ( $p=0.064$ and 0.057 , respectively; Table 2 ). Additionally, worse OS and DFS were observed in patients with higher METTL3 expression in both well-differentiated histologic grade (G1) ( $p=0.002$ and 0.002 , respectively; Table 2$)$ and moderately/poorly histologic grade (G2-3) $(p<0.001$ and $p<0.001$, respectively; Table 2). METTL3 expression showed no statistically significant relationship with histopathology tumor status or histological grade by the clinicopathologic analysis (Table 1). The sample size, different grouping and analysis methods might affect the statistical results of the relationship between METTL3 expression and histopathology tumor status or histological grade.

Consistent results were found in the subgroup analysis of TNM stage. Worse OS and DFS were observed in patients with higher METTL3 expression in both the early stage subgroup (TNM stage I+II) $(p=0.025$ and 0.02 , respectively; Figure $2 \mathrm{~B}$ ) and the advanced stage subgroup (TNM stage III) $(p=0.002$ and 0.001 , respectively; Figure 2C). Similar results were observed in the histopathology subgroups of $\mathrm{pT} 3-4, \mathrm{pN}=0, \mathrm{pN}=1 / 2 / 3, \mathrm{G} 1$ and G2-3 (Table 2).

\section{METTL3 Expression Level Was an Independent Predictor for DFS and OS in ESCC Patients}

Using cox proportional hazard model, we performed univariate and multivariate survival analysis to explore the effect of METTL3 expression on OS or DFS of ESCC. As presented in Table 3, the univariate survival analysis revealed that nodal status $(\mathrm{HR}=3.040,95 \% \mathrm{CI}: 2.063-4.481, p<0.001)$ and METTL3 expression level (HR $=2.389,95 \%$ CI: 1.652 3.454, $p<0.001)$ are predictors for DFS of ESCC patients. Multivariate survival analysis also showed similar results with HR of $2.932(95 \%$ CI: $1.983-4.335, p<0.001)$ for 
A

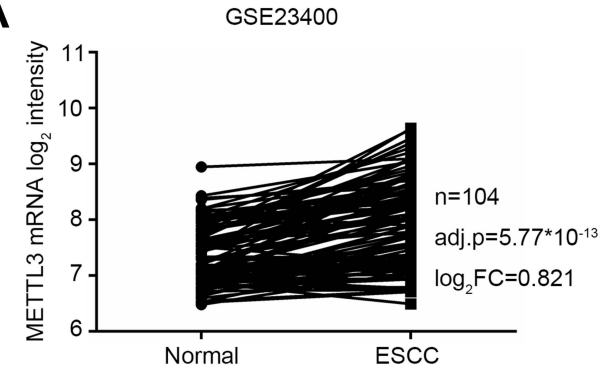

C

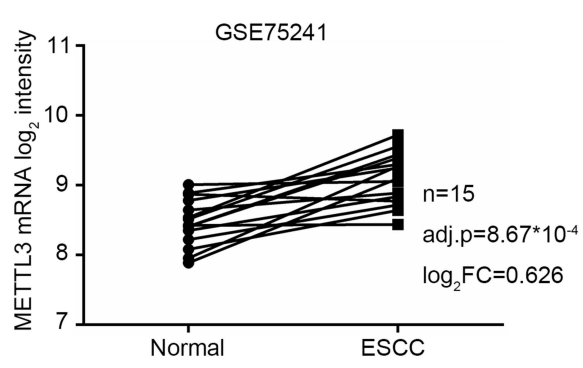

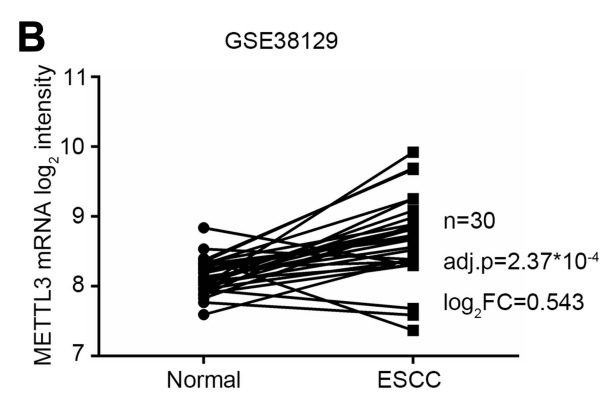
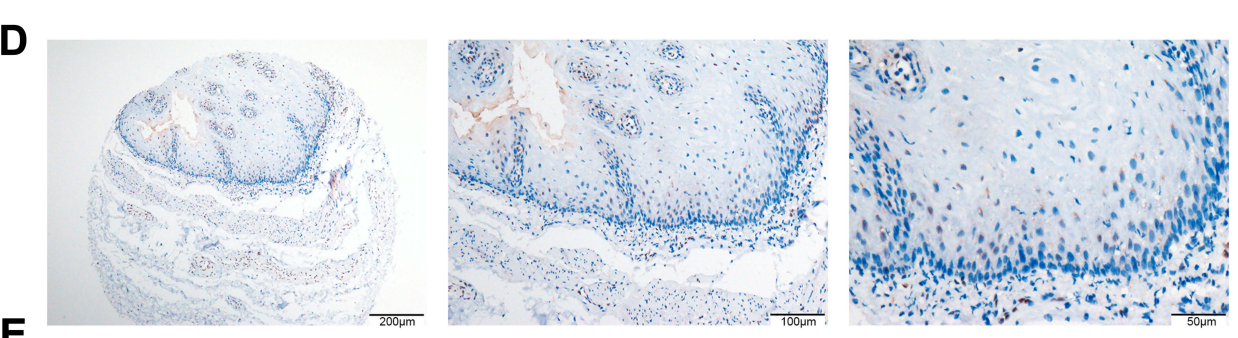

E

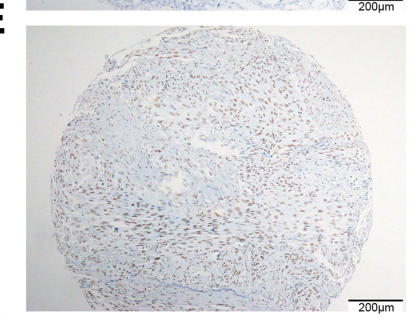

$\mathbf{F}$
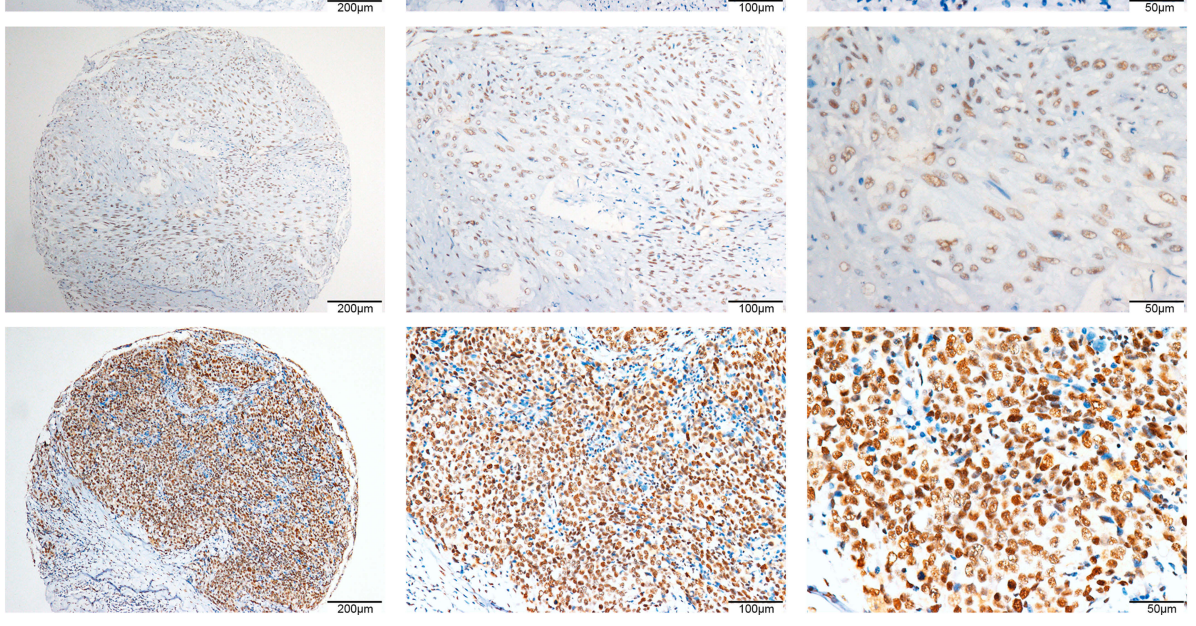

Figure I The METTL3 expression in ESCC normal and tumor tissue.

Notes: (A-C) The mRNA expression levels of METTL3 in ESCC tumor and matched normal samples analyzed by three open access microarray data, GSE23400 (A), GSE38I29 (B) and GSE7524I (C). Data are significant at adj.p < 0.05. (D-F) The results of immunohistochemistry assay (IHC) in normal esophageal mucosa (D), in ESCC tumor with METTL3 low expression (E) and METTL3 high expression (F).

Abbreviations: adj.p, adjusted $p$-value calculated by the Benjamini-Hochberg approach for the false discovery rate; ESCC, esophageal squamous cell carcinoma; log ${ }_{2} \mathrm{FC}$, $\log _{2}$ transformation of fold change of METTL3 expression; METTL3, methyltransferase like 3.

nodal status ( $\mathrm{N}>0)$ and HR of 2.264 (95\% CI: 1.563-3.281, $p<0.001)$ for METTL3 high expression. We also performed univariate and multivariate survival analysis for OS of ESCC. As presented in Table 4, the univariate survival analysis revealed higher histopathology tumor status ( $\mathrm{HR}=1.503,95 \% \mathrm{CI}: 1.014-2.227, p=0.043)$, lymph nodepositive $(\mathrm{HR}=3.170,95 \% \mathrm{CI}: 2.150-4.673, p<0.001)$ and high METTL3 expression level $(\mathrm{HR}=2.356,95 \%$ CI: 
Table I METTL3 Expression and Clinicopathologic Variables of 207 ESCC Cases

\begin{tabular}{|c|c|c|c|c|}
\hline \multirow[t]{2}{*}{ Variables } & \multirow[t]{2}{*}{ Cases $(n=207)$} & \multicolumn{2}{|l|}{ METTL3 Expression } & \multirow[t]{2}{*}{$p$-value ${ }^{a}$} \\
\hline & & Low $(n=128$, percent $)$ & High ( $n=79$, percent) & \\
\hline Age $^{b}$ (years) & & & & 0.855 \\
\hline$\leq 58$ & 111 & $68(53.1)$ & $43(54.4)$ & \\
\hline$>58$ & 96 & $60(46.9)$ & $36(45.6)$ & \\
\hline Sex & & & & 0.905 \\
\hline Female & 56 & $35(27.3)$ & $2 \mathrm{I}(26.6)$ & \\
\hline Male & $|5|$ & $93(72.7)$ & $58(73.4)$ & \\
\hline Tumor location & & & & 0.456 \\
\hline Upper & 12 & $9(7.0)$ & $3(3.8)$ & \\
\hline Middle & 140 & $83(64.8)$ & $57(72.2)$ & \\
\hline Lower & 55 & $36(28.1)$ & $19(24.1)$ & \\
\hline Histological grade ${ }^{c}$ & & & & 0.279 \\
\hline Well differentiated (GI) & 51 & $27(2 I . I)$ & $24(30.4)$ & \\
\hline Moderately differentiated (G2) & $|3|$ & $86(67.2)$ & $45(57)$ & \\
\hline Poorly differentiated (G3) & 25 & $15(11.7)$ & $10(12.7)$ & \\
\hline PT status ${ }^{c}$ & & & & 0.157 \\
\hline pTI & 6 & $5(3.9)$ & $\mathrm{I}(\mathrm{I} .3)$ & \\
\hline pT2 & 47 & $34(26.6)$ & $13(16.5)$ & \\
\hline pT3 & 151 & $88(68.8)$ & $63(79.7)$ & \\
\hline $\mathrm{pT} 4$ & 3 & $\mathrm{I}(0.8)$ & $2(2.5)$ & \\
\hline$N$ categories $^{c}$ & & & & $0.045^{*}$ \\
\hline No & 110 & $75(58.6)$ & $35(44.3)$ & \\
\hline$N>0$ & 97 & $53(4 I .4)$ & $44(55.7)$ & \\
\hline TNM stage $^{c}$ & & & & $0.004^{*}$ \\
\hline Stage I & 7 & $4(3.1)$ & $4(3.8)$ & \\
\hline Stage II & 119 & $85(66.4)$ & $34(43)$ & \\
\hline Stage III & 81 & $39(30.5)$ & $42(53.2)$ & \\
\hline
\end{tabular}

Notes: ${ }^{*} p$-value $<0.05$ indicates statistical significance. ${ }^{a} p$-values were calculated by Pearson's correlation test. ${ }^{b}$ Age is classified according to the median age of 58-year-old. 'The grading and histopathology stage of ESCC specimens are based on the World Health Organization classification published in 2009. Abbreviations: ESCC, esophageal squamous cell carcinoma; METTL3, methyltransferase like 3; N categories, lymph node metastasis status; N0, lymph node metastasis negative; $\mathrm{N}>0$, lymph node metastasis positive; $\mathrm{PT}$ status, histopathology tumor status; TNM stage, tumor/node/metastasis staging classification.

1.629-3.407, $p<0.001)$ are associated with poor OS Multivariate analysis indicated that nodal status $(\mathrm{HR}=3.078,95 \% \mathrm{CI}: 2.080-4.555, p<0.001)$ and METTL3 expression level $(\mathrm{HR}=2.164,95 \% \mathrm{CI}: 1.487-3.151, p<0.001)$ are predictors for OS of ESCC patients. Here, the univariate survival analysis revealed that histopathology tumor status are associated with $\mathrm{OS}(\mathrm{HR}=1.503,95 \% \mathrm{CI}: 1.014-2.227$, $p=0.043$, Table 4) and nearly associated with DFS (HR=1.417, 95\% CI: 0.957-2.099, $p=0.082$, Table 3). Multivariate analysis indicated that histopathology tumor status was not recognized as an independent prognostic factor of OS and DFS. The sample size might affect the statistical analysis of histopathology tumor status.
These data demonstrated that the expression level of METTL3 was significantly related to the clinicopathological characteristics of the ESCC patients and is an efficient predictor of DFS and OS for ESCC patients. Altogether, METTL3 appears to be a good predictive factor for ESCC patients, supporting its potential clinical application as a prognostic biomarker for ESCC.

\section{Discussion}

In this study, we found that METTL3 was upregulated in ESCC, and its expression level might be a good predictive factor for ESCC patients, supporting its potential clinical application as a prognostic biomarker for ESCC. 
Table 2 METTL3 Expression in ESCC Patients by Kaplan-Meier Survival Analysis (Log Rank Test)

\begin{tabular}{|c|c|c|c|c|c|c|c|}
\hline \multirow[t]{2}{*}{ Variables } & \multirow[t]{2}{*}{ Cases } & \multicolumn{3}{|c|}{ DFS (months) } & \multicolumn{3}{|c|}{ OS (months) } \\
\hline & & Mean & Median & $p$-value & Mean & Median & $p$-value \\
\hline \multicolumn{8}{|l|}{ Total } \\
\hline Low expression & 128 & 72.4 & NR & $<0.00 I^{*}$ & 74.9 & NR & $<0.00 I^{*}$ \\
\hline High expression & 79 & 38.6 & 18 & & 44 & 25 & \\
\hline \multicolumn{8}{|l|}{ pT status ${ }^{\mathrm{a}}$} \\
\hline \multicolumn{8}{|l|}{ PTI-2 } \\
\hline Low expression & 39 & 66.5 & NR & 0.064 & 70.1 & NR & 0.057 \\
\hline High expression & 14 & 42.3 & 30 & & 48.5 & 54 & \\
\hline \multicolumn{8}{|l|}{ PT3-4 } \\
\hline Low expression & 89 & 72.3 & NR & $<0.00 I^{*}$ & 74.1 & NR & $<0.00 I^{*}$ \\
\hline High expression & 65 & 37.2 & 17 & & 42.1 & 25 & \\
\hline \multicolumn{8}{|l|}{$\mathrm{N}$ categories ${ }^{\mathrm{a}}$} \\
\hline \multicolumn{8}{|l|}{ No } \\
\hline Low expression & 75 & 86.4 & NR & $0.006 *$ & 88.1 & NR & $0.009 *$ \\
\hline High expression & 35 & 58.6 & 58 & & 63.2 & 66 & \\
\hline \multicolumn{8}{|l|}{$N>0$} \\
\hline Low expression & 53 & 44.2 & 29 & $<0.00 I^{*}$ & 48.3 & 40 & $<0.00 I^{*}$ \\
\hline High expression & 44 & 19.6 & 10 & & 25.7 & 15 & \\
\hline \multicolumn{8}{|l|}{ Histologic grade ${ }^{a}$} \\
\hline \multicolumn{8}{|l|}{ GI } \\
\hline Low expression & 27 & 74.6 & NR & $0.002 *$ & 76.4 & NR & $0.002^{*}$ \\
\hline High expression & 24 & 37.5 & 26 & & 44 & 45 & \\
\hline \multicolumn{8}{|l|}{ G2-3 } \\
\hline Low expression & 101 & 69.7 & NR & $<0.00 I^{*}$ & 72.4 & 74 & $<0.00 I^{*}$ \\
\hline High expression & 55 & 38.1 & 14 & & 43 & 25 & \\
\hline \multicolumn{8}{|l|}{ TNM stage $^{\mathrm{a}}$} \\
\hline \multicolumn{8}{|l|}{ Stage I+II } \\
\hline Low expression & 89 & 83 & NR & $0.02^{*}$ & 85.2 & NR & $0.025^{*}$ \\
\hline High expression & 37 & 60 & 58 & & & & \\
\hline \multicolumn{8}{|l|}{ Stage III } \\
\hline Low expression & 39 & 40 & 27 & $0.001 *$ & 43.2 & 39 & $0.002 *$ \\
\hline High expression & 42 & 17.2 & 9 & & 23.1 & 15 & \\
\hline
\end{tabular}

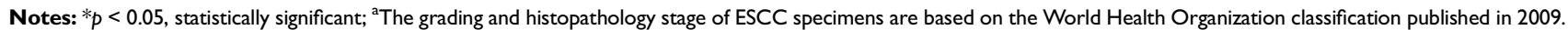
Abbreviations: DFS, disease-free survival; ESCC, esophageal squamous cell carcinoma; GI, well differentiated; G2-3, moderately differentiated and poorly differentiated; METTL3, methyltransferase like 3; $\mathrm{N}$ categories, lymph node metastasis status; N0, lymph node metastasis negative; $\mathrm{N}>0$, lymph node metastasis positive; NR, not reached; OS, overall survival; PT status, histopathology tumor status; TNM stage, tumor/node/metastasis staging classification.

The $\mathrm{m}^{6} \mathrm{~A}$ writer METTL3 is a core catalytic component of methyltransferase complex. Recent studies have shown that METTL3 could regulate various tumors' progression via $\mathrm{m}^{6} \mathrm{~A}$ modification. For example, METTL3 plays oncogenic function in breast cancer, liver cancer, acute myeloid leukemia, glioblastoma or others. ${ }^{19-22}$ However, the expression status of METTL3 in ESCC was not characterized so far. Given that ESCC accounts for about $90 \%$ of esophageal cancers in Asia, with a particularly high prevalence in Eastern to Central Asia, it is worth to investigate its role involved in ESCC development and progression.

In this study, we at first identify the expression status of METTL3 in ESCC at the RNA and protein level. We 

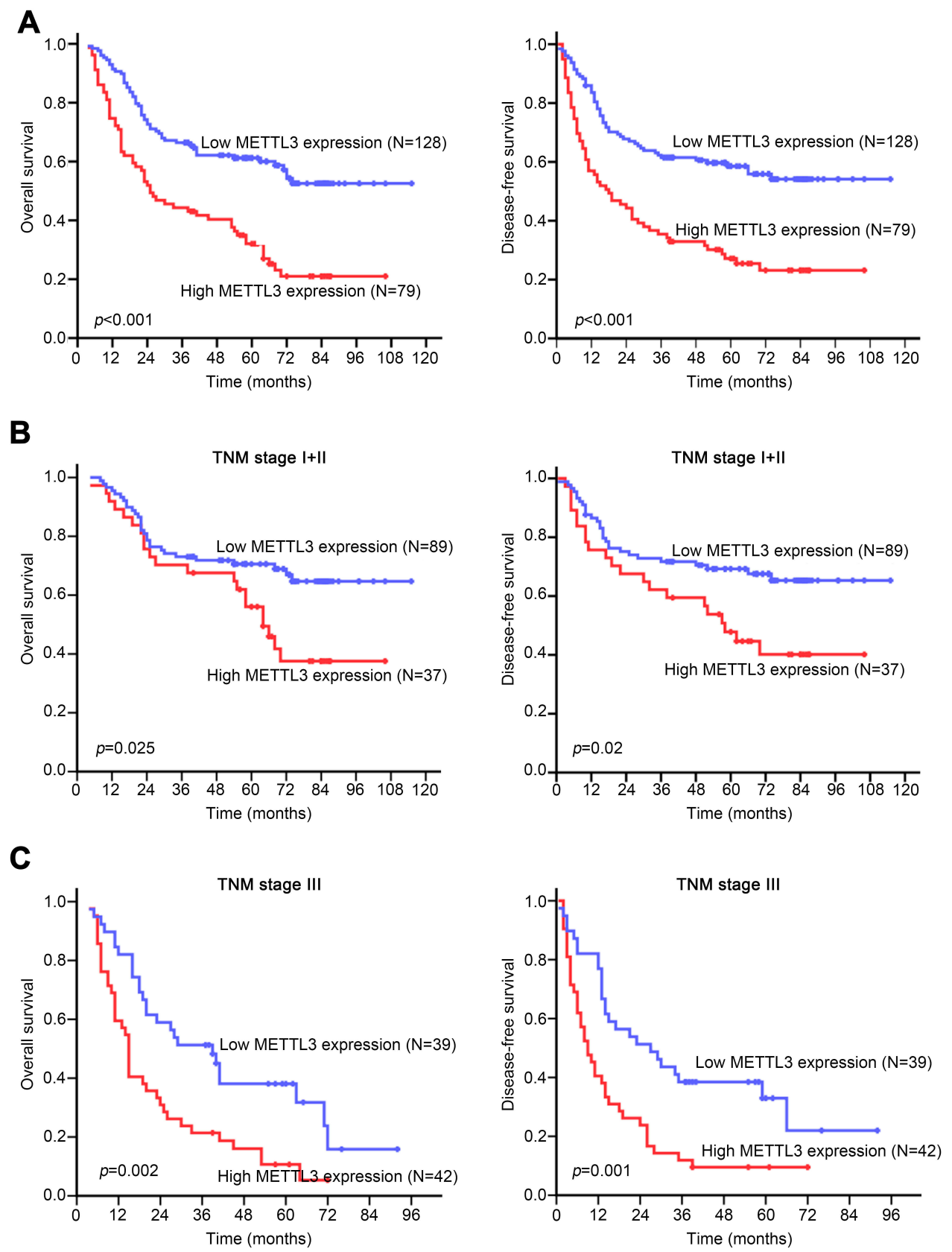

Figure 2 High METTL3 expression was associated with poor survival.

Notes: (A) The Kaplan-Meier plots of OS and DFS for 207 ESCC patients classified according to their METTL3 expression level. $p<0.05$, statistically significant. (B) The Kaplan-Meier plots of OS and DFS for 126 ESCC patients in TNM stage I+II classified according to their METTL3 expression level. $p<0.05$, statistically significant. (C) The Kaplan-Meier plots of OS and DFS for 8I ESCC patients in TNM stage III classified according to their METTL3 expression level. p<0.05, statistically significant.

Abbreviations: DFS, disease-free survival; ESCC, esophageal squamous cell carcinoma; METTL3, methyltransferase like 3; OS, overall survival; TNM stage, tumor/node/ metastasis staging classification.

analyzed open available datasets of ESCC and found that mRNA level of METTL3 was upregulated in ESCC tissues compared with adjacent nonmalignant esophageal mucosal tissues. We also performed IHC assays and found that METTL3 was higher expressed in cancer cell of the ESCC tissues. According to the expression level of METTL3, the IHC samples were classified into two subgroups: high and low expression of METTL3. Higher expression level of METTL3 was associated with worse OS and DFS in all samples and TNM stage I+II subgroup or TNM stage III subgroup. After prognostic analysis, we found that high expression of METTL3 was associated with $\mathrm{N}$ categories, TNM stages and poor prognosis. Using univariate and multivariate survival analysis, we 
Table 3 Univariate and Multivariate Analysis of Disease-Free Survival of ESCC Patients

\begin{tabular}{|c|c|c|c|c|c|c|}
\hline \multirow[t]{2}{*}{ Variables } & \multicolumn{3}{|c|}{ Univariate Analysis } & \multicolumn{3}{|c|}{ Multivariate Analysis } \\
\hline & HR & $(95 \% \mathrm{Cl})$ & p-value & HR & $(95 \% \mathrm{Cl})$ & $p$-value \\
\hline Age $^{a}$ (years) $(>58$ vs $\leq 58)$ & 0.984 & $0.68 I-1.421$ & 0.930 & & & \\
\hline Gender (male vs female) & 1.066 & $0.706-1.611$ & 0.761 & & & \\
\hline Tumor location (upper/middle/lower) & 0.833 & $0.582-1.192$ & 0.319 & & & \\
\hline Histological grade ${ }^{b}(G 3 / G 2 / G I)$ & 1.213 & $0.888-1.657$ & 0.225 & & & \\
\hline pT status ${ }^{b}$ (pT4/pT3/pT2/pTI) & 1.417 & $0.957-2.099$ & 0.082 & & & \\
\hline $\mathrm{N}$ categories $^{\mathrm{b}}(\mathrm{N}>0 / \mathrm{N} 0)$ & 3.040 & $2.063-4.481$ & $<0.001 *$ & 2.932 & $1.983-4.335$ & $<0.00 I^{*}$ \\
\hline METTL3 expression (High/Low) & 2.389 & $1.652-3.454$ & $<0.00 I^{*}$ & 2.264 & $|.563-3.28|$ & $<0.00 I^{*}$ \\
\hline
\end{tabular}

Notes: ${ }^{*} p<0.05$, statistically significant. ${ }^{\text {a }}$ Age is classified according to the median age of 58 -year-old. ${ }^{\text {b}}$ The grading and histopathology stage of ESCC specimens are based on the World Health Organization classification published in 2009.

Abbreviations: ESCC, esophageal squamous cell carcinoma; GI, well differentiated; G2, moderately differentiated; G3, poorly differentiate; HR, hazard ratio; METTL3, methyltransferase like 3; $\mathrm{N}$ categories, lymph node metastasis status; N0, lymph node metastasis negative; N>0, lymph node metastasis positive; pT status, histopathology tumor status; $95 \% \mathrm{Cl}$, the $95 \%$ confidence interval.

Table 4 Univariate and Multivariate Analysis of Overall Survival of ESCC Patients

\begin{tabular}{|c|c|c|c|c|c|c|}
\hline \multirow[t]{2}{*}{ Variables } & \multicolumn{3}{|c|}{ Univariate Analysis } & \multicolumn{3}{|c|}{ Multivariate Analysis } \\
\hline & HR & $(95 \% \mathrm{Cl})$ & p-value & HR & $(95 \% \mathrm{Cl})$ & p-value \\
\hline Age $^{\mathrm{a}}$ (years) (>58 vs. $\left.\leq 58\right)$ & 0.980 & $0.678-1.416$ & 0.914 & & & \\
\hline Gender (male vs female) & 1.120 & $0.741-1.692$ & 0.591 & & & \\
\hline Tumor location (upper/middle/lower) & 0.862 & $0.600-1.239$ & 0.423 & & & \\
\hline Histological grade ${ }^{\mathrm{b}}(\mathrm{G} 3 / \mathrm{G} 2 / \mathrm{GI})$ & 1.190 & $0.872-1.624$ & 0.272 & & & \\
\hline $\mathrm{pT}$ status $^{\mathrm{b}}$ (pT4/pT3/pT2/pTI) & 1.503 & $1.014-2.227$ & $0.043 *$ & 1.312 & $0.879-1.960$ & 0.184 \\
\hline $\mathrm{N}$ categories $^{\mathrm{b}}(\mathrm{N}>0 / \mathrm{N} 0)$ & 3.170 & $2.150-4.673$ & $<0.001^{*}$ & 3.078 & $2.080-4.555$ & $<0.00 I^{*}$ \\
\hline METTL3 expression (High/Low) & 2.356 & I.629-3.407 & $<0.00 I^{*}$ & 2.164 & $|.487-3.15|$ & $<0.00 I^{*}$ \\
\hline
\end{tabular}

Notes: ${ }^{*} p<0.05$, statistically significant. ${ }^{\mathrm{a}} \mathrm{Age}$ is classified according to the median age of 58-year-old. ${ }^{\mathrm{b}}$ The grading and histopathology stage of ESCC specimens are based on the World Health Organization classification published in 2009.

Abbreviations: ESCC, esophageal squamous cell carcinoma; GI, well differentiated; G2, moderately differentiated; G3, poorly differentiate; HR, hazard ratio; METTL3, methyltransferase like 3; $\mathrm{N}$ categories, lymph node metastasis status; N0, lymph node metastasis negative; N>0, lymph node metastasis positive; $\mathrm{PT}$ status, histopathology tumor status; $95 \% \mathrm{Cl}$, the $95 \%$ confidence interval.

found that the expression level of METTL3 was an independent factor on DFS and OS of ESCC.

Therefore, our results indicated that METTL3 was more highly expressed in ESCC. The increased expression of METTL3 was significantly positively associated with poor prognosis compared with low METTL3 phenotype. After analyzing of clinicopathological factors, METTL3 expression level was an independent predictor for DFS and OS of ESCC patients. Collectively, our results reveal that the METTL3 expression level could be used as an independent prognostic biomarker for ESCC patient survival. However, the mechanisms of METTL3 in ESCC progression remain to investigate by further studies.

\section{Data Sharing Statement}

The authenticity of this article has been validated by uploading the key raw data onto the Research Data Deposit public platform (www.researchdata.org.cn), with the approval RDD number as RDDB2020000861. 


\section{Author Contributions}

All authors made substantial contributions to conception and design, acquisition of data, or analysis and interpretation of data; took part in drafting the article or revising it critically for important intellectual content; gave final approval of the version to be published; and agree to be accountable for all aspects of the work.

\section{Funding}

This study was supported by the National Natural Science Foundation of China $(81830090,81802775)$, Natural Science Foundation of Guang Dong Province (No. 20181025201938243).

\section{Disclosure}

The authors report no conflicts of interest in this work.

\section{References}

1. Wei CM, Gershowitz A, Moss B. 5'-Terminal and internal methylated nucleotide sequences in HeLa cell mRNA. Biochemistry. 1976;15 (2):397-401. doi:10.1021/bi00647a024

2. Desrosiers R, Friderici K, Rottman F. Identification of methylated nucleosides in messenger RNA from Novikoff hepatoma cells. Proc Natl Acad Sci U S A. 1974;71(10):3971-3975. doi:10.1073/ pnas.71.10.3971

3. Meyer KD, Saletore Y, Zumbo P, Elemento O, Mason CE, Jaffrey SR. Comprehensive analysis of mRNA methylation reveals enrichment in 3' UTRs and near stop codons. Cell. 2012;149(7):1635-1646. doi:10.1016/j.cell.2012.05.003

4. Dominissini D, Moshitch-Moshkovitz S, Schwartz S, et al. Topology of the human and mouse m6A RNA methylomes revealed by m6A-seq. Nature. 2012;485(7397):201-206. doi:10.1038/nature11112

5. Edupuganti RR, Geiger S, Lindeboom RGH, et al. N6methyladenosine (m6A) recruits and repels proteins to regulate mRNA homeostasis. Nat Struct Mol Biol. 24:870-878. doi:10.1038/ nsmb.3462

6. Schwartz S, Mumbach MR, Jovanovic M, et al. Perturbation of m6A writers reveals two distinct classes of mRNA methylation at internal and 5' sites. Cell Rep. 2014;8(1):284-296. doi:10.1016/j.celrep.2014.05.048

7. Ping XL, Sun BF, Wang L, et al. Mammalian WTAP is a regulatory subunit of the RNA N6-methyladenosine methyltransferase. Cell Res. 2014;24(2):177-189. doi:10.1038/cr.2014.3

8. Bokar JA, Shambaugh ME, Polayes D, Matera AG, Rottman FM Purification and cDNA cloning of the AdoMet-binding subunit of the human mRNA (N6-adenosine)-methyltransferase. Rna. 1997;3 (11):1233-1247.
9. Liu J, Yue Y, Han D, et al. A METTL3-METTL14 complex mediates mammalian nuclear RNA N6-adenosine methylation. Nat Chem Biol. 2014;10(2):93-95. doi:10.1038/nchembio.1432

10. Jia G, Fu Y, Zhao X, et al. N6-methyladenosine in nuclear RNA is a major substrate of the obesity-associated FTO. Nat Chem Biol. 2011;7(12):885-887. doi:10.1038/nchembio.687

11. Zheng G, Dahl JA, Niu Y, et al. ALKBH5 is a mammalian RNA demethylase that impacts RNA metabolism and mouse fertility. Mol Cell. 2013;49(1):18-29. doi:10.1016/j.molcel.2012.10.015

12. Shi H, Wang X, Lu Z, et al. YTHDF3 facilitates translation and decay of N6-methyladenosine-modified RNA. Cell Res. 2017;27 (3):315-328. doi:10.1038/cr.2017.15

13. Li A, Chen YS, Ping XL, et al. Cytoplasmic m(6)A reader YTHDF3 promotes mRNA translation. Cell Res. 2017;27(3):444-447. doi:10.1038/cr.2017.10

14. Wang X, Lu Z, Gomez A, et al. N6-methyladenosine-dependent regulation of messenger RNA stability. Nature. 2014;505 (7481):117-120. doi:10.1038/nature 12730

15. Wang X, Zhao BS, Roundtree IA, et al. N(6)-methyladenosine modulates messenger RNA translation efficiency. Cell. 2015;161 (6):1388-1399. doi:10.1016/j.cell.2015.05.014

16. Xiao W, Adhikari S, Dahal U, et al. Nuclear m(6)A reader YTHDC1 regulates mRNA splicing. Mol Cell. 2016;61(4):507-519. doi:10.1016/j.molcel.2016.01.012

17. Wojtas MN, Pandey RR, Mendel M, Homolka D, Sachidanandam R, Pillai RS. Regulation of m(6)A transcripts by the $3^{\prime}->5^{\prime}$ RNA Helicase YTHDC2 is essential for a successful meiotic program in the mammalian germline. Mol Cell. 2017;68(2):374-387. (). doi:10.1016/j.molcel.2017.09.021

18. Hsu PJ, Zhu Y, Ma H, et al. Ythde2 is an N(6)-methyladenosine binding protein that regulates mammalian spermatogenesis. Cell Res. 2017;27(9):1115-1127. doi:10.1038/cr.2017.99

19. Chen M, Wei L, Law CT, et al. RNA N6-methyladenosine methyltransferase METTL3 promotes liver cancer progression through YTHDF2 dependent post-transcriptional silencing of SOCS2. Hepatology. 2017.

20. Cai X, Wang X, Cao C, et al. HBXIP-elevated methyltransferase METTL3 promotes the progression of breast cancer via inhibiting tumor suppressor let-7g. Cancer Lett. 2017;415:11-19. doi:10.1016/j. canlet.2017.11.018

21. Visvanathan A, Patil V, Arora A, et al. Essential role of METTL3-mediated m6A modification in glioma stem-like cells maintenance and radioresistance. Oncogene. 2017.

22. Vu LP, Pickering BF, Cheng Y, et al. The N(6)-methyladenosine ( $\mathrm{m}$ (6)A)-forming enzyme METTL3 controls myeloid differentiation of normal hematopoietic and leukemia cells. Nat Med. 2017;23 (11):1369-1376. doi:10.1038/nm.4416

23. Abbas G, Krasna M. Overview of esophageal cancer. Ann Cardiothorac Surgery. 2017;6(2):131-136. doi:10.21037/ acs.2017.03.03

24. Zhao J, He YT, Zheng RS, Zhang SW, Chen WQ. Analysis of esophageal cancer time trends in China, 19892008. Asian Pacific j Cancer Prev. 2012;13(9):4613-4617. doi:10.7314/APJCP.2012.13.9.4613

25. Jemal A, Siegel R, Xu J, Ward E. Cancer statistics, 2010. CA Cancer J Clin. 2010;60(5):277-300. doi:10.3322/caac.20073
Cancer Management and Research is an international, peer-reviewed open access journal focusing on cancer research and the optimal use of preventative and integrated treatment interventions to achieve improved outcomes, enhanced survival and quality of life for the cancer patient.
The manuscript management system is completely online and includes a very quick and fair peer-review system, which is all easy to use. Visit http://www.dovepress.com/testimonials.php to read real quotes from published authors. 